\title{
Biojardineras como alternativas para el tratamiento de aguas residuales: experiencia en cinco biojardineras en las comunidades de Barra Honda y La Vigía de Nicoya, Guanacaste ${ }^{1}$
}

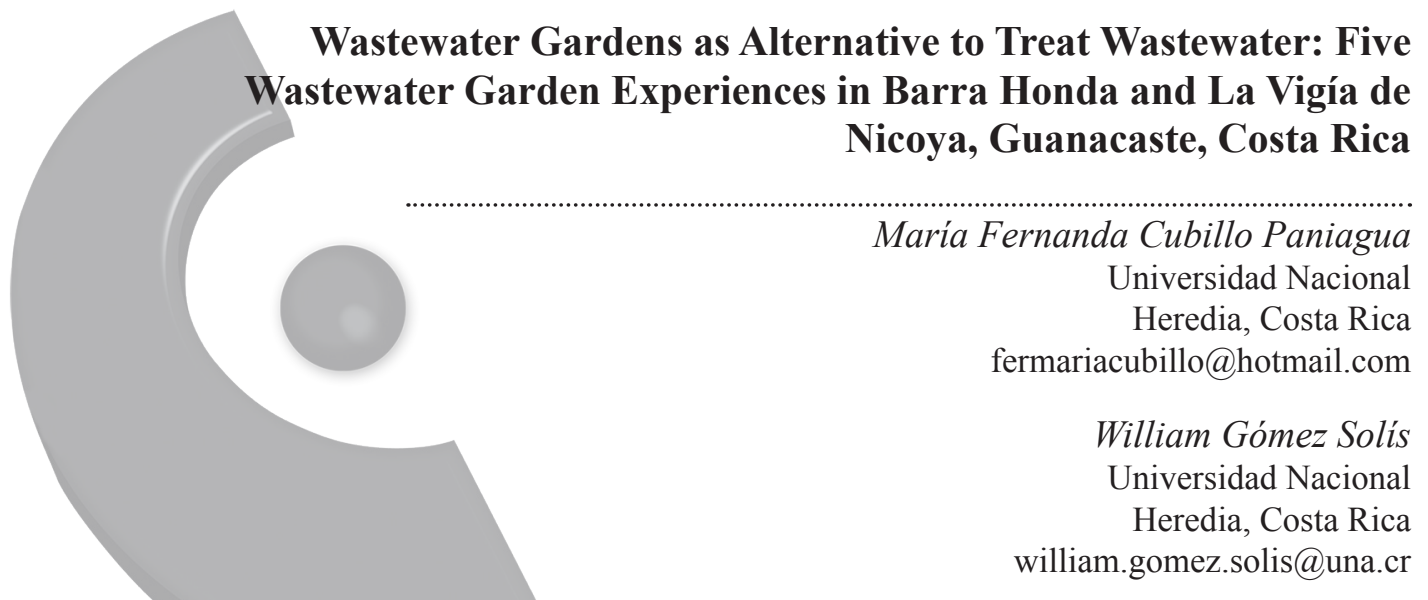

Recibido: 23/11/2016 Aceptado 18/04/2017

Resumen: El presente trabajo busca promocionar la biojardinera como un sistema eficiente de tratamiento de aguas residuales de bajo costo, a partir de los beneficios obtenidos en la implementación de cinco de estas como producto del proyecto Mejoramiento en el tratamiento de las aguas residuales a través de biojardinerasen las comunidades de Barra Honda y La Vigía, del cantón de Nicoya, Guanacaste, con el propósito de informar, crear conciencia y despertar el interés de iniciativas de personas, instituciones o a nivel país que contribuyan a la investigación, sensibilización y desarrollo de las biojardineras, para de esta forma con-

Agradecimientos a la Msc. Silvia Lorena Zúñiga Guerrero, silvialo2007@yahoo.es; la Msc. Maritza Marín Araya,maritza5cr@gmail. com; al Lic. Jairo Guevara Rosales, jairo.guevara.rosales@una.cr; la Lic. Yendri Vargas, yendryvatre@gmail.com; la Msc. Nelly López Alfaro,nlopez@una.cr; al Lic. Didier Gómez, dgomez.sae@ice.co. cr; y al Dr. Mario William Acosta Cortez, macostacortes@gmail.com 
tinuar avanzando en la articulación de esfuerzos desde lo local en el ámbito institucional y comunitario para el mejoramiento en la salud pública y los ecosistemas naturales. Zúñiga (2015) menciona que "ante la escasa atención e importancia que se está dando en Costa Rica al tratamiento de las aguas residuales tanto en el ámbito normativo, en su práctica y en su supervisión siguen siendo el reto más importante para administrar el riesgo de contaminación de los cuerpos de agua" (p. 5).

Palabras claves: agua, biojardinera, salud pública, contaminación, participación ciudadana.

\begin{abstract}
The present work seeks to promote wastewater gardens as an efficient system of low-cost treatment for sewage water. This intention came up from the benefits obtained with the implementation of five of these methods as part of a project conducted in Barra Honda and La Vigía de Nicoya, Guanacaste, Costa Rica. In fact, the project "Improvement in the treatment of sewage water through wastewater gardens" was implemented with the purpose of informing individuals, institutions and the country about these systems, and raising awareness of and interest in initiatives that encourage research efforts, awareness raising and development of these methods, and thus, to continue advancing in the articulation of efforts from the institutional and community level in order to improve the public health and the natural ecosystems. Zuñiga (2015) says that "due to little attention and importance given to the treatment of sewage water in Costa Rica in the regulatory field, in its practice and in its supervision, this process remains the most important challenge in order to manage the pollution risk of water bodies."
\end{abstract}

Keywords: water, wastewater garden, public health, pollution, citizen participation.

\title{
Introducción
}

El Pacífico Norte de Costa Rica se caracteriza por poseer una época seca y una lluviosa bien definidas, sin embargo en los últimos años, debido al cambio climático, el periodo de lluvia en todo el país, pero principalmente en esta región, ha ido en detrimento, lo cual ha causado estragos en el sector agropecuario, y en algunos cantones la disponibilidad del agua potable comienza a presentar problemas.

El agua es un factor fundamental para la sostenibilidad, por su importancia para la vida humana, las actividades productivas y la integridad ecológica. En los últimos años el problema de disponibilidad y calidad hídrica ha aumentado y se ha convertido en una discusión sobre los derechos humanos, la equidad social y el equilibrio ambiental. El Programa Estado de la Nación (2016) menciona que: 
El país exhibe logros importantes en el acceso y la cobertura de agua potable, pero existen varias situaciones que atentan contra la posibilidad de mantenerlos. En primer lugar, se carece de información suficiente para conocer y manejar el recurso, lo cual incide en la capacidad de planificar su uso, una tarea urgente en un marco de cambio climático y alta contaminación. Y en segundo lugar, la lentitud de los avances en materia de tratamiento y saneamiento significa una amenaza, por su impacto sobre los cuerpos de agua, escasamente protegidos (p. 224).

Y aunque parezca que la cobertura de tratamiento a nivel nacional es alta, existen grandes problemas que según el Ministerio de Planificación Nacional y Política Económica (2010)

Se relacionan con las dos principales opciones de tratamiento y disposición final del país, y es que la mayoría de los sistemas de alcantarillado no cuenta con un sistema de tratamiento, por lo que sirven solamente para transportar las aguas residuales a su destino final, que en la mayoría de los casos son los ríos, donde los lodos generados en los sistemas de tanque séptico se evacúan y su disposición final también es en los ríos (p. 117).

Es por ello que se promueve la búsqueda de sistemas de saneamiento de bajo costo "que puedan ser desarrollados en forma privada y adaptados a las condiciones existentes del entorno relacionados con condiciones del suelo, clima, planificación urbana, prácticas culturales de la población, entre otros" (Moncada, 2011, citado por Zúñiga, 2015).

Ante este panorama, durante el periodo 2015-2016 se construyeron cinco biojardineras, tres en la comunidad de Barra Honda y dos en la comunidad de La Vigía, ambas del cantón de Nicoya, de Guanacaste, como producto del proyecto Mejoramiento en el tratamiento de aguas residuales a través de biojardineras. Este proyecto se desarrolló en el Centro Mesoamericano de Desarrollo Sostenible del Trópico Seco (Cemede), apoyado por el programa Horizontes Ambientales del Instituto de Estudios de la Población (Idespo) de la Universidad Nacional de Costa Rica, apoyado también por el Ministerio de Salud de Nicoya.

Después de haberse visto ejecutadas las cuatro etapas del proyecto anteriormente mencionado (diseño y/o formulación del proyecto, sensibilización antes de la construcción, construcción de las biojardineras y manteni- 
miento de los sistemas) es el momento oportuno para identificar e informar sobre el impacto y beneficios que trajo la implementación de las biojardineras en el ámbito social, económico y ambiental en las comunidades donde se desarrolló esta iniciativa y de esta forma promocionar esta tecnología, no como una solución cabal al problema del mal manejo de las aguas residuales que está ocurriendo en nuestro país, pero sí como una alternativa eficiente de saneamiento de bajo costo que pueda ser desarrollada en forma privada individual o comunal y adaptada a las condiciones existentes del entorno relacionadas con condiciones del suelo, clima, planificación urbana, prácticas culturales de la población, entre otras.

\section{Estado de la cuestión}

En Costa Rica el reto del siglo XX fue lograr la cobertura de agua a nivel residencial, ahora el gran reto del presente siglo es tratar las aguas ya utilizadas. "Diferentes estudios realizados en el país sostienen que el porcentaje de agua tratada no supera el $5 \%$ del total utilizado para el año 2013” (CGR, DFOE-AE-IF-01-2013, citado por Zúñiga, 2015).

A los problemas de escasez de agua producto del cambio climático que cada año va en aumento se suma el mal manejo de las aguas residuales, esto a causa de muchos factores, como la falta de experiencia por parte de la población y las instituciones para implementar tecnologías alternativas para el manejo de estas aguas, la falta de sensibilización que existe en la población, la resistencia al cambio de viejos hábitos, entre muchos otros. Donde la combinación de estas dos situaciones acelera el deterioro de los ecosistemas presentes en los cuerpos de agua.

Como una primera aproximación en el país actualmente se han empezado a implementar sistemas de tratamiento de aguas grises a nivel de residencias denominados biojardineras, impulsadas principalmente por la Asociación Centroamericana para la Economía, la Salud y el Ambiente (Acepesa) en conjunto con otras entidades como el Instituto Tecnológico de Costa Rica, Asadas y el Ministerio de Agricultura y Ganadería.

Dentro de las acciones inmediatas para la atención de la gestión sostenible de agua ante la sequía y acceso de agua a las poblaciones y producción en la vertiente Pacífico Norte, el presidente de la república, el ministro de Ambiente y Energía, el ministro de Agricultura y Ganadería y el ministro de Turismo mediante el Decreto 38642-MP-MAG señalan lo siguiente: 
Declaró emergencia nacional la situación generada por la sequía que afectó los cantones de Liberia, Tilarán, Nicoya, Santa Cruz, Bagaces, Carrillo, Cañas, Abangares, Nandayure, La Cruz y Hojancha de la provincia de Guanacaste, los cantones de Aguirre, Garabito, Montes de Oro, Esparza y cantón central de la provincia de Puntarenas y los cantones de Orotina, San Mateo y Atenas, de la provincia de Alajuela (p. 2).

Las políticas públicas, leyes, decretos y/o acuerdos tienen su enorme grado de importancia, sin embargo, si la población tuviera mayor conciencia de estas problemáticas, podría unir esfuerzos y ayudar a disminuir los riesgos provocados a la salud y al ambiente por el manejo inadecuado de las aguas residuales.

\section{Marco teórico}

\section{Recurso hídrico}

El agua es un factor clave para la sostenibilidad por sus implicaciones para la vida humana, las actividades productivas y la integridad ecológica. Según el Programa Estado de la Nación (2015):

En los últimos años el debate sobre este recurso ha trascendido la sola preocupación por su disponibilidad y calidad, para convertirse en una discusión sobre los derechos humanos, la equidad social y el equilibrio ambiental. El país exhibe logros importantes en el acceso y la cobertura de agua potable, pero existen varias situaciones que atentan contra la posibilidad de mantenerlos. En primer lugar, se carece de información suficiente para conocer y manejar el recurso, lo cual incide en la capacidad de planificar su uso, una tarea urgente en un marco de cambio climático y alta contaminación. Y en segundo lugar, la lentitud de los avances en materia de tratamiento y saneamiento significa una amenaza, por su impacto sobre los cuerpos de agua, escasamente protegidos.

Aunque el acceso al agua potable sigue en aumento $(93,4 \%$ en 2014), la disponibilidad comienza a presentar problemas: en algunos cantones se han paralizado actividades de construcción por dificultades para garantizar el abastecimiento del líquido.

La cobertura de alcantarillado sanitario con tratamiento de aguas residuales pasó de 3,6\% a 4,2\%, luego de casi diez años de no presentar variaciones (p. 183). 
Revista Universidad en Diálogo • Vol. 7, N. ${ }^{\circ}$ 1, Enero-Junio 2017, pp. 69-87

ISSN 2215-2849 • EISSN: 2215-4752

DOI: http://dx.doi.org/10.15359/udre.7-1.4

\section{Aguas residuales}

Según la Iniciativa Integrada para un Ambiente Urbano Sostenible (2006):

Las aguas grises o residuales son aquellas aguas que se producen en nuestra casa, en la escuela, en el trabajo y en todo lugar donde se use agua con fines de limpieza o de higiene de nuestros cuerpos, ropa y utensilios, pero, sin llevar las "excretas" (orines y heces). Estas aguas grises salen de las pilas para lavar ropa, del baño, del lavamanos, del fregadero de la cocina y de otros usos domésticos. Esas aguas contienen diversos contaminantes del tipo orgánico y del tipo nutrientes (compuestos de nitrógeno y fósforo). Pudiendo entonces contener bacterias como también elementos que con procedimientos sencillos podrán recuperarse y reutilizarse (p. 5).

Cuando las aguas residuales no son tratadas adecuadamente para mejorar su calidad, provocan un impacto negativo en el ambiente, estimulando la creación de focos de contaminación y enfermedades, "provocando malos olores, y reproduciendo vectores transmisores del dengue, chikungunya, zika, por mencionar los más conocidos por la población. Las enfermedades transmitidas por vectores representan más del 17\% de todas las enfermedades infecciosas, y provocan cada año más de 1 millón de defunciones" (OMS, 2016).

\section{Biojardinera}

La biojardinera "es un humedal artificial, utilizada como técnica alternativa para el tratamiento de las aguas grises que hasta hace poco se viene presentando, su atractivo consiste en contar con una jardinera que da belleza a nuestra casa que al mismo tiempo mejora la calidad de esas aguas" (Zúñiga, 2015, p. 3).

Es un sistema vivo, ya que en él interactúan los componentes biológicos contenidos en las aguas residuales con los elementos del sistema, tales como raíces de las plantas, la piedra y la velocidad a la que el agua circula dentro del mismo. Todos ellos hacen que el sistema realice el proceso de purificación del agua.

Una vez limpia, se puede reutilizar en el riego de jardines, patios, en usos agropecuarios, entre otros. También infiltrándola por medio de un drenaje o descargándola en algún curso natural que exista en las cercanías. También con las plantas sembradas se pueden hacer arreglos florales o artesanías, entre otras actividades. 
En resumen la tecnología de limpieza del agua está compuesta por tres elementos principales.

a. El Tratamiento primario o pretratamiento.

b. La biojardinera y su mantenimiento.

c. El depósito o aprovechamiento de las aguas tratadas (Gómez, 2016, p. 3).

\section{Sensibilización}

"Es el aumento o generación de capacidades a una población determinada, con el fin de modificar y/o adaptar nuevas prácticas a las formas de vida y de trabajo, ante circunstancias o panoramas que antes no existían o no eran muy conocidos" (Dirección General de Medio Ambiente del Gobierno de Navarra, 2002, p. 30).

La sensibilización se genera a partir de espacios de participación e integración entre actores que deseen contribuir al desarrollo de un territorio, con la orientación y apoyo de un equipo de trabajo compuesto por especialistas de diferentes campos del conocimiento y de unidades que nutren su proceso de aprendizaje, aportando conocimientos en el entendimiento de la problemática.

La idea principal es sensibilizar a un grupo de la población, que adquiera un compromiso real de prácticas que logren convertirse en hábitos para mejorar, cambiar o mitigar las causas y efectos de algunas problemáticas.

En la experiencia desarrollada en el proyecto Mejoramiento en el tratamiento de aguas residuales a través de biojardineras en el cantón de Nicoya, antes de la instalación de las biojardineras, se sensibilizó a las comunidades de Barra Honda y La Vigía, en las cuales se ejecutó el proyecto a través de la generación de capacidades en funcionarios institucionales y a nivel familiar en el uso de tecnologías de tratamiento de aguas grises domiciliares de bajo costo, como lo son las biojardineras, con el fin de que esa modalidad pueda ser utilizada como una opción de salud ambiental y saneamiento local en las comunidades para una mejor calidad de vida de sus habitantes. 
Revista Universidad en Diálogo • Vol. 7, N. ${ }^{\circ}$ 1, Enero-Junio 2017, pp. 69-87

ISSN 2215-2849 • EISSN: 2215-4752

DOI: http://dx.doi.org/10.15359/udre.7-1.4

\section{Salud pública}

En relación con este tema Vargas y Piedrola (2009) mencionan que:

Es la ciencia y el arte de organizar y dirigir los esfuerzos colectivos destinados a proteger, promover y restaurar la salud de los habitantes, su desarrollo se caracteriza en la responsabilidad colectiva de la salud, debe ser dirigida a la población, manteniendo énfasis en la prevención, unido a la atención de los factores de riesgo y a los determinantes socioeconómicos de la salud (p. 3).

La práctica de la salud pública debe basarse en evidencias concisas de toma de decisiones, planteamientos, políticas, análisis de programas de salud, a nivel país, institucional y local, para poder cumplir su objetivo.

Según la Organización Panamericana de la Salud (2009):

Entre las enfermedades de declaración obligatoria para el año 2007, las que se presentan con mayor frecuencia están la diarrea (4.952,9 casos por 100.000 habitantes), el dengue (con 583,4 casos por 100.000 habitantes), la gonorrea (con 30,8 casos), el paludismo (con 27,2 casos), la hepatitis (con 23,3 casos), la tos ferina y tuberculosis (con 11 y 11,5 casos respectivamente) (p. 4).

En el cantón de Nicoya, de acuerdo con datos suministrados por el Área Rectora de Salud de Nicoya, "las enfermedades transmitidas por vectores en el periodo 2013 para el cantón de Nicoya según orden de incidencia son: iras (20.881 casos), diarreas (3.010 casos), dengue (2.859 casos)" (Ministerio de Salud, 2013, citado por Zúñiga, 2015).

La biojardinera es una alternativa que ayuda a disminuir muchas de las anteriores enfermedades, ya que elimina los problemas de estancamiento de aguas; reduce la contaminación de los cuerpos de aguas superficiales y subterráneas; reduce la presión sobre el agua potable al reutilizar fuentes de mayor calidad en labores que actualmente demandan agua potable; y mejora el paisaje escénico.

\section{Seguridad alimentaria}

Según la Organización de las Naciones Unidas para la Agricultura y la Alimentación (FAO), desde la Cumbre Mundial de la Alimentación (CMA) de 1996, 
La seguridad alimentaria a nivel de individuo, hogar, nación y global, se consigue cuando todas las personas, en todo momento, tienen acceso físico y económico a suficiente alimento, seguro y nutritivo, para satisfacer sus necesidades alimenticias y sus preferencias, con el objeto de llevar una vida activa y sana.

Desde sus inicios, las Naciones Unidas han establecido el acceso a una alimentación adecuada como derecho individual y responsabilidad colectiva. La Declaración Universal de Derechos Humanos de 1948 proclamó que "toda persona tiene derecho a un nivel de vida adecuado que le asegure, así como a su familia, la salud y el bienestar, y en especial la alimentación".

Aunado a esto se encuentra la soberanía alimentaria, que según el Programa Especial para la Seguridad Alimentaria (2011)

Es el derecho de los pueblos, las naciones o las uniones de países a definir sus políticas agrícolas y de alimentos, sin ningún dumping frente a, países terceros. La soberanía alimentaria organiza la producción y el consumo de alimentos acorde con las necesidades de las comunidades locales, otorgando prioridad a la producción para el consumo local y doméstico (p. 3).

Nuestra sociedad enfrenta en estos momentos grandes desafíos para la sobrevivencia por el aumento de los precios de los alimentos, pero lamentablemente las soluciones no se visualizan en el corto plazo, entonces cada uno de los esfuerzos que contribuyen a buscar respuestas inmediatas ante esta serie de problemas resultan meritorios.

\section{Belleza escénica}

Este concepto conlleva aspectos subjetivos, pero ligados a la conservación y el disfrute de un patrimonio heredado, porque está constituido por una amplia gama de recursos naturales, como ríos, montañas, volcanes, lagos, bosques y la biodiversidad, los cuales tienen un significativo valor económico que pocas veces es reconocido por la población. El Instituto Interamericano de Cooperación para la Agricultura (2010) menciona que:

Aun cuando se han efectuado estimaciones de valoración económica relacionada con la belleza escénica, los resultados son muy preliminares y no permiten conclusiones precisas al respecto, sobre todo si se toma en cuenta que no hay experiencia acumulada en la transacción de estos recursos en el mercado de bienes y servicios (p. 30). 
La degradación ambiental por la transformación del entorno natural ha afectado la calidad de la belleza escénica de los paisajes como resultado del proceso de desgaste de grandes áreas de suelo, bosques, cuencas y la contaminación visual. El Instituto Interamericano de Cooperación para la Agricultura (2010) señala que:

En los últimos años se ha tratado de revertir la tendencia antes citada, con políticas de carácter ambiental principalmente expresadas en la consolidación del Sistema de Parques Nacionales y Áreas Protegidas. Entre los efectos positivos de estas medidas destacan, entre otros, el rescate de la valiosa riqueza biológica nacional, la preservación de cuencas, la restauración de áreas forestales y la gradual toma de conciencia en cuanto al reconocimiento del paisaje como una fuente de recursos que presta un servicio ambiental de alto valor para la recreación de la población y el desarrollo en especial del ecoturismo (p. 10).

Es necesario mejorar la calidad del paisaje escénico mediante la aplicación de diferentes mecanismos identificados por el Estado como el Pago de Servicios Ambientales (PSA). Este esfuerzo debe contar con la participación decidida de grupos comunales organizados, las municipalidades e instituciones públicas involucrados en el desarrollo ambiental del país.

\section{Metodología}

La metodología del trabajo se basó en la participación que se obtuvo en el proyecto Mejoramiento en el tratamiento de las aguas residuales a través de biojardineras por medio de las experiencias adquiridas en la implementación de cinco biojardineras, tres en la comunidad de Barra Honda y dos en La Vigía, del cantón de Nicoya, cuya ejecución se logró a través de la producción del conocimiento propositivo, construido a partir del debate, la reflexión y la construcción colectiva de saberes entre diferentes actores de un territorio en busca de la transformación social.

La investigación-acción-participación se inició a partir de una acción, que al ser evaluada a través de la experiencia por los propios participantes proporcionó reflexiones para entender el proceso y encontrar otras alternativas posibles de solución con el objetivo de mejorar el conocimiento de dicha práctica.

Los principales actores involucrados en el proyecto fueron:

- Autoridades y funcionarios del área rectora del Ministerio de Salud de Nicoya que tienen dentro de sus funciones el saneamiento ambiental. 
- Instituto de Estudios de la Población (Idespo) de la Universidad Nacional de Costa Rica

- Centro Mesoamericano de Desarrollo Sostenible del Trópico Seco (Cemede) de la Universidad Nacional de Costa Rica

Por medio de la identificación de los beneficios sociales, ambientales y económicos que se obtuvieron a nivel individual, comunal e institucional con la ejecución del proyecto, se elaboró el presente artículo científico con el objetivo de promocionar este sistema para el tratamiento de las aguas residuales, y de esta forma crear conciencia y despertar el interés de iniciativas de personas, instituciones o a nivel país que contribuyan a la investigación, sensibilización y desarrollo de las biojardineras.

\section{Resultados}

En la primera fase del proyecto se realizó una matriz con la colaboración del Área Rectora de Salud de Nicoya para seleccionar las familias en las comunidades de Barra Honda y La Vigía, con el fin de seleccionar las que por condiciones pudieran manejar una biojardinera y hacerse cargo del mantenimiento periódicamente. Este proceso se vio acompañado de talleres de sensibilización y de la presentación del manejo de las aguas grises por medio de las biojardineras, dando a conocer a los participantes el diseño, la función y el manejo de las biojardineras. Una vez seleccionadas las familias, se procedió a la construcción de las estructuras, tres en la comunidad de Barra Honda y dos en La Vigía.

Posterior a la construcción de las biojardineras está la necesidad de darle el seguimiento y la supervisión a las estructuras, además de la sensibilización de las familias y la comunidad que ha participado en el proceso en temas ambientales como la separación de residuos, manejo de desechos, entre otros. Se insiste en el adecuado mantenimiento del sistema y surge la iniciativa de reutilizar las aguas tratadas en el sistema de la biojardinera, y una opción es la implementación de huertas familiares. El manejo de las biojardineras pasó de ser un sistema para el tratamiento de aguas residuales a ser un elemento que abarca una amplia gama de beneficios a nivel familiar, como: ornato en los patios, disminución de malos olores, eliminación de vectores como zancudos, adaptación y mitigación al cambio climático, salud mental y seguridad alimentaria. 
Revista Universidad en Diálogo • Vol. 7, N. ${ }^{\circ}$ 1, Enero-Junio 2017, pp. 69-87

ISSN 2215-2849 • EISSN: 2215-4752

DOI: http://dx.doi.org/10.15359/udre.7-1.4

Tabla 1

Costos generales de biojardinera modelo, 2016

\begin{tabular}{lcrc}
\hline Detalle & Costos por metro $(\not \subset)$ & Total $(\not \subset)$ & $\%$ \\
\hline Costos Materiales & 36.983 & 443.795 & $38 \%$ \\
Mano DE Obra & 14.694 & 176.333 & $15 \%$ \\
$\begin{array}{l}\text { Servicios Técnicos } \\
\text { (biojardinera) }\end{array}$ & 45.000 & 540.000 & $47 \%$ \\
$\begin{array}{l}\text { Transporte Vagoneta (15 } \\
\text { metros cúbicos) }\end{array}$ & 5.000 & 60.000 & $5 \%$ \\
Transporte vehículo rural & & & \\
\hline Total & 850 & 12.750 & $1 \%$ \\
\hline
\end{tabular}

Nota: ASEPESA, 2016.

Los costos de los materiales se estimaron a partir del modelo estándar diseñado por Acepesa para Barra Honda de 4x12 metros. En tanto los costos por servicios técnicos corresponden al costo por unidad de un bloque de cuatro biojardineras construidas a la vez.

Los costos de transporte se presentan por kilómetro, considerando que es un costo representativo de acuerdo con la ubicación de las biojardineras con relación al punto de venta de la piedra.

Los costos de mano de obra se estimaron a partir del conteo de horas de la mano de obra comunal aportada a un precio de 1500 colones la hora. Aunque este costo es contrapartida familiar, se estimó para considerar el costo real de la biojardinera. Incluye el costo de los servicios de un fontanero o albañil a un costo de 2000 colones la hora.

La tabla 2 muestra los registros del consumo de agua en las viviendas con biojardineras desde el mes de mayo del 2015 hasta octubre del 2016, estos datos fueron obtenidos con la información presente en la base de datos del Instituto Costarricense de Acueductos y Alcantarillados con el número de usuario del servicio de cada vivienda.

Estos análisis fueron realizados por el Centro de Recursos Hídricos para Centroamérica y el Caribe en mayo del 2016, cada análisis fue basado en los métodos estándar para el análisis de aguas y aguas residuales de la Asociación Americana de Salud Pública, de la Asociación Americana del Agua y la Federación Ambiental del Agua en su edición veintidós publicada en el 2012. 
Revista Universidad en DiÁlogo • Vol. 7, N. ${ }^{\circ} 1$, Enero-Junio 2017, pp. 69-87

ISSN 2215-2849 • EISSN: 2215-4752

DOI: http://dx.doi.org/10.15359/udre.7-1.4

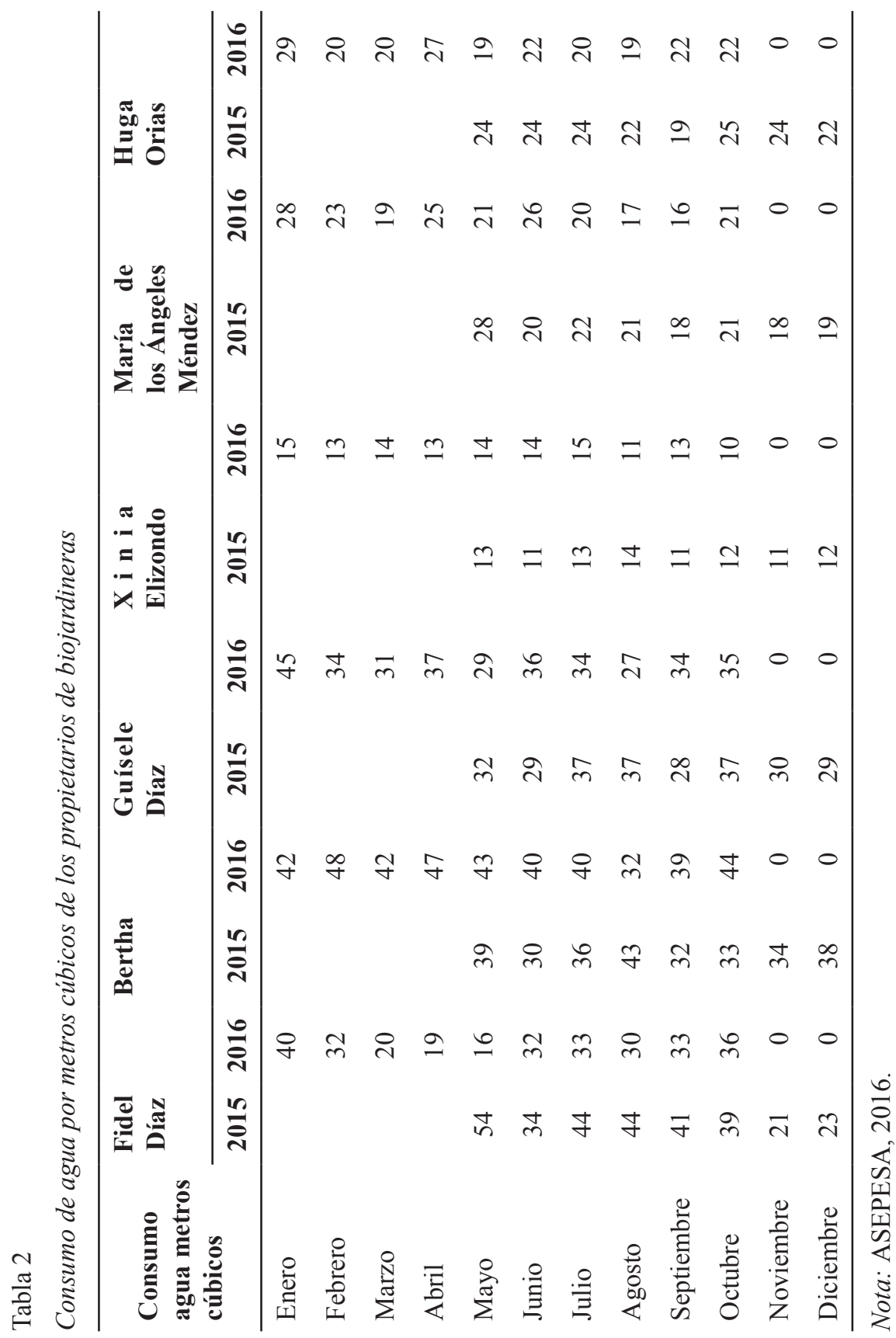


Revista Universidad en Diálogo • Vol. 7, N. ำ Enero-Junio 2017, pp. 69-87

ISSN 2215-2849 • EISSN: 2215-4752

DOI: http://dx.doi.org/10.15359/udre.7-1.4

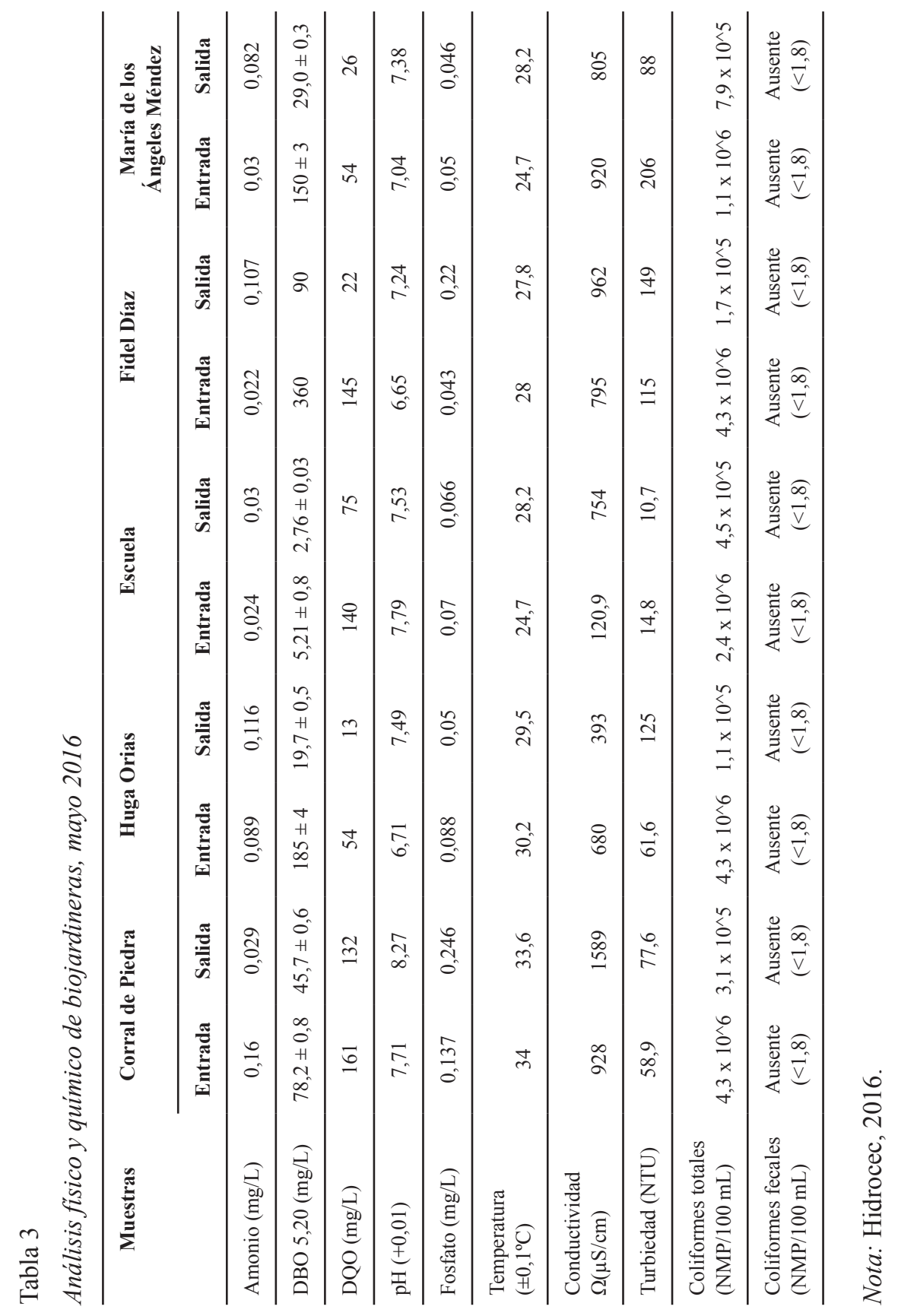




\section{Discusión}

Fue importante y necesario sensibilizar a las personas de las comunidades, pero principalmente a las familias seleccionadas, antes de la construcción de los sistemas, ya que de esta forma se forjó un compromiso real con la estructura en relación con el funcionamiento, mantenimiento del sistema, capacidad de transmitir sus conocimientos a otras personas y aprovechamiento del agua tratada, para de esta forma garantizar el éxito de la ejecución del proyecto.

Es importante recalcar que en el proceso, por situaciones personales dentro de las familias, se presentaron casos en los que los sistemas quedaron sin el debido mantenimiento en algunos periodos, además, que el nivel del compromiso con respecto al manejo de las estructuras no ha sido igual en todos los casos. Una de las principales razones es el tema cultural, por ejemplo, solo se le daba mantenimiento si había conocimiento de que la estructura iba a ser monitoreada o visitada por alguna entidad, no en todos los casos, pero sí fue una tendencia encontrada durante el proceso.

El constante monitoreo y el acompañamiento fueron reforzando el nivel de compromiso en cuanto al manejo adecuado de las estructuras. Otro elemento que colaboró en este aspecto fue el intercambio de experiencias con la comunidad de San Antonio de Atenas, en la cual funciona una biojardinera comunal que da tratamiento a siete hogares. Las aguas son reutilizadas en cultivos agrícolas, principalmente en cultivos de café. Este intercambio de experiencias reforzó la importancia del mantenimiento, donde uno de los principales puntos a tratar fue la colaboración de todos los miembros del hogar que tienen biojardineras, incentivando el manejo participativo para que toda la responsabilidad no recaiga sobre una sola persona.

Entre los beneficios indirectos que se detectaron con el establecimiento de las biojardineras, se encuentra el elemento de la salud mental, ya que en todos los casos los propietarios expresaban que el darle mantenimiento a la estructura los ayudaba a distraerse del entorno, incluso les funcionaba como terapia para aclarar los pensamientos o simplemente distraerse de los problemas cotidianos y la rutina, principalmente cuando se manejaban las plantas de la biojardinera, elemento que en la mayoría de casos llena de ilusión a las mujeres que manejan la biojardinera por el tema del embellecimiento de sus patios.

Además, está el tema de los mosquitos o zancudos que, según la percepción general, han disminuido considerablemente desde el funcionamiento de las estructuras, elemento de suma importancia considerando que la región Cho- 
Revista Universidad en Diálogo • Vol. 7, N. ำ Enero-Junio 2017, pp. 69-87

ISSN 2215-2849 • EISSN: 2215-4752

DOI: http://dx.doi.org/10.15359/udre.7-1.4

rotega es uno de los sectores con más incidencia de casos de dengue transmitidos por el mosquito Aedes aegypti y el panorama actual de las enfermedades del zika y el chikungunya transmitidas por este mismo mosquito.

A nivel familiar la implementación de las biojardineras es un elemento que controla la propagación de criaderos, principalmente por medio del ornato y limpieza de los patios y los alrededores de los hogares donde se encuentran las estructuras.

Dentro de los beneficios que brinda la biojardinera como sistema de tratamiento de las aguas residuales estaba pendiente el tema de qué hacer con el agua una vez tratada, por lo que plantea la necesidad de reutilizar las aguas tratadas. En una primera etapa se estaban utilizando para regar las plantas ornamentales en los alrededores de las viviendas, pero la cantidad de agua residual era suficiente para ser implementada en otros usos. De esta manera, por iniciativa propia los dueños establecieron una pequeña huerta cerca de la biojardinera y la regaban con el agua residual; otra de las familias la utilizaba en la limpieza de una pequeña porqueriza y en abastecer un abrevadero para dos vacas. Se promueve entonces la implementación de pequeñas huertas para que cada familia a pequeña escala produzca diferentes hortalizas o legumbres que les ayuden en su alimentación diaria.

De acuerdo con los costos para la construcción de una biojardinera (tabla 1), la inversión que se requiere es considerada alta para las familias de clase media o baja. A pesar de no ser un sistema accesible para toda la población de forma individual por la inversión que esta requiere, sí es posible su construcción por medio de instituciones que ejecutan trabajos de investigación con comunidades, o iniciativas colectivas comunales que tengan interés de llevar a cabo estos sistemas, que sean conscientes del gran aporte que estarían realizando al medio ambiente y a sus vidas individuales particularmente.

Con respecto al consumo de agua en las familias (tabla 2) no se ha podido establecer un marco de referencia que determine con exactitud la disminución en el consumo del agua por familia, dado que se detectan problemas de fugas en algunos hogares, lo que afecta la comparación. Por otro lado, está el entorno familiar en el que algunos casos presentan unas condiciones en las que es inevitable el uso del agua potable en mayor medida por cuestiones de higiene y salud. Pero a nivel personal cada familia percibe que sí disminuyen el consumo de agua potable por la utilización de las aguas de las biojardineras, ya que reutilizan estas aguas, principalmente en actividades de riego. 
Sobre los análisis de las muestras de agua de las biojardineras, es importante recalcar que al momento de estos análisis en la mayoría de los casos estos sistemas llevaban poco tiempo de estar en funcionamiento y no han alcanzado el máximo potencial en el tratamiento de las aguas residuales; a pesar de esto, se encuentran resultados importantes para determinar la eficacia del sistema en el tratamiento de las aguas grises de los hogares. Uno de los parámetros más importantes es la eliminación de contaminantes, ya que en los valores de la demanda biológica de oxígeno (DBO), demanda química de oxígeno (DQO), amonio, fosfatos y la presencia de coliformes hay una considerable disminución de agentes patógenos o elementos que indican el grado de contaminación del agua, formación de microorganismos u oxidación del agua. Esto pensado en el contexto de que estas aguas sean vertidas en cuerpos de agua como quebradas o ríos, con menor contaminación que en el caso de ser vertidas directamente sin el tratamiento previo.

En indicadores como el $\mathrm{pH}$ y la conductividad por la relativa "juventud" de los sistemas y por el tipo de material utilizado en la construcción estos valores incrementan; en el caso del $\mathrm{pH}$, que se refiere al grado de acidez o qué tan alcalina es el agua, se encuentra que aumenta, manteniéndose en valores alcalinos. La turbiedad no presenta valores tan significativos porque se encontró que el material rocoso implementado desprendió partículas, lo que puede afectar el paso de luz en las muestras tomadas. Pero a pesar de que estas aguas no alcanzan niveles de pureza, en lo que se puedan implementar para consumo sí demuestran que este tipo de sistemas son efectivos para el tratamiento de las aguas residuales, principalmente en las regiones rurales donde este tema no ha sido muy manejado por las diferentes instituciones.

\section{Conclusiones}

La implementación de estas estructuras como una opción a nivel familiar, vecinal o comunal implica un proceso que va más allá de solo construir la biojardinera, se requiere un gran criterio para la selección de las personas que pueden hacerse cargo del mantenimiento de la estructura, además del fomento en la adquisición de hábitos de higiene que garanticen el buen funcionamiento de todo el sistema. Una vez alcanzados estos cambios en los hábitos familiares y que exista un compromiso adecuado en el seguimiento y mantenimiento de la estructura se puede ver el apropiamiento sobre la estructura en las familias. 
En un principio se determinaron las biojardineras como un medio para tratar aguas residuales, pero se ha determinado que las mismas implican una serie de beneficios a nivel familiar en la parte de ornato de los patios, seguridad alimentaria, control de vectores, disminución en el recibo de agua, disminución en la contaminación ambiental y mejora de la salud mental.

Se demuestra que estos sistemas efectivamente son una opción viable en el tratamiento de aguas residuales, ya que a pesar de que no han alcanzado su máximo potencial en el funcionamiento debido a que son muy jóvenes, los resultados de los análisis presentan valores considerables en la eliminación de contaminantes y carga de partículas dañinas en el agua.

\section{Referencias}

Asociación Centroamericana para la Economía, la Salud y el Ambiente (Acepesa). (2006). Creando jardines para limpiar nuestra agua. Manual para la construcción de biojardineras. San Jose, Costa Rica: Acepesa.

Asociación Centroamericana para la Economía, la Salud y el Ambiente (Acepesa). (2016). Servicios profesionales para desarrollar acciones en el marco del proyecto Mejoramiento en el tratamiento de aguas residuales a través de biojardineras. San Jose, Costa Rica: Acepesa.

Centro de Recursos Hidrológicos para Centroamérica y el Caribe (Hidrocec). (2016). Reporte de resultados. Versión 001. Consecutivo RR023-2016. Universidad Nacional de Costa Rica, Sede Regional Chorotega, Liberia, Costa Rica.

Dirección General de Medio Ambiente del Gobierno de Navarra (2006). Manual Módulo de Sensibilización Ambiental ( $2^{\mathrm{a}}$ ed.). Unión Europea.

Gómez, W. (2016). Biojardineras y cosecha de agua de lluvia (reservorios y sistema de purificación). Centro Mesoamericano de Desarrollo Sostenible del Trópico Seco (Cemede). Nicoya, Costa Rica, Universidad Nacional.

Instituto Interamericano de Cooperación para la Agricultura (IICA). (2010). Un nodo de cooperación técnica sobre los servicios ambientales en Costa Rica. Oficina del IICA en Costa Rica. División de Cooperación Técnica Horizontal. Cartago, Costa Rica. 
La salud y sus determinantes. Concepto de medicina preventiva y salud pública. (2009). En G. Piédrola, Medicina preventiva y salud pública (10 ed.). Barcelona, España: Masson.

Ministerio de Ambiente y Energía (Minae) (2016). Decreto DAJ-D-0402016-Minae. Acciones Inmediatas para la Atención de la Gestión Sostenible de Agua ante la Sequía y Acceso de Agua a las Poblaciones y Producción en la Vertiente Pacífico Norte. Recuperado de http://www.minae.go.cr/recursos/2016/pdf/MINAE-Propuesta-Decreto-N40-2016Acciones-Sequia-ConsultaWEBSET2016.pdf

Ministerio de Planificación Nacional y Política Económica. (2010). Costa Rica: objetivos de desarrollo del milenio, II Informe país. San José, Costa Rica.

Organización Mundial de la Salud (OMS). (2016). Nota descriptiva Nº 387, en el Centro de Prensa sobre Enfermedades Transmitidas por Vectores. Recuperado de http://www.who.int/mediacentre/factsheets/ fs $387 /$ es/

Organización Panamericana de la Salud (OPS) / Organización Mundial de la Salud (OMS). (2009). Perfil del sistema de salud de Costa Rica. Monitoreo y análisis de los procesos de cambio y reforma. Washington, Estados Unidos.

Programa Estado de la Nación en Desarrollo Humano Sostenible (PEN). (2015). Vigésimo primer informe estado de la nación en desarrollo humano sostenible/PEN. San José, Costa Rica: PEN.

Programa Estado de la Nación en Desarrollo Humano Sostenible (PEN). (2016). Vigésimo segundo informe estado de la nación en desarrollo humano sostenible/PEN. San José, Costa Rica: PEN.

Programa Especial para la Seguridad Alimentaria-PESA (2011). Seguridad alimentaria. Conceptos básicos. Proyecto Food Facility, Honduras.

Zúñiga, S. (2015). Proyecto Mejoramiento en el tratamiento de aguas residuales a través de biojardineras. Sistema de Información Académica (SIA). Formulación de proyecto académico. Sede Regional Chorotega, Universidad Nacional. Nicoya, Costa Rica. 